OPEN ACCESS

Edited by:

Xiaolong Ji,

Zhengzhou University of Light Industry, China

Reviewed by:

Yanglei Yi,

Northwest A\&F University, China Hongshun Yang,

National University of

Singapore, Singapore

*Correspondence:

Daodong Pan

daodongpan@163.com

Specialty section:

This article was submitted to Food Chemistry

a section of the journal

Frontiers in Nutrition

Received: 09 October 2021 Accepted: 06 December 2021 Published: 04 January 2022

Citation:

Fan X, Li X, Zhang T, Guo Y, Shi Z, Wu Z, Zeng $X$ and Pan D (2022) Novel Millet-Based Flavored Yogurt Enriched With Superoxide Dismutase.

Front. Nutr. 8:791886. doi: 10.3389/fnut.2021.791886

\section{Novel Millet-Based Flavored Yogurt Enriched With Superoxide Dismutase}

\author{
Xiankang Fan 1,2, Xiefei $\mathrm{Li}^{1,2}$, Tao Zhang ${ }^{3}$, Yuxing Guo ${ }^{3}$, Zihang Shi ${ }^{1,2}$, Zhen $\mathrm{Wu}^{1,2}$, \\ Xiaoqun Zeng ${ }^{1,2}$ and Daodong Pan ${ }^{1,2 *}$
}

${ }^{1}$ Key Laboratory of Animal Protein Food Processing Technology of Zhejiang Province, College of Food and Pharmaceutical Sciences, Ningbo University, Ningbo, China, ${ }^{2}$ State Key Laboratory for Managing Biotic and Chemical Threats to the Quality and Safety of Agro-Products, Ningbo University, Ningbo, China, ${ }^{3}$ School of Food Science and Pharamaceutical Engineering, Nanjing Normal University, Nanjing, China

Superoxide dismutase (SOD) is an important antioxidant enzyme with different physiological functions, which can be used as a nutritional fortifier in food. Cereal-based fermented products are becoming popular worldwide. In this study, novel millet-based flavored yogurt enriched with SOD was developed. Lactiplantibacillus plantarum subsp. plantarum was screened, which manufactured SOD activity of $2476.21 \pm 1.52 \mathrm{U} \mathrm{g}^{-1}$. The SOD content of millet yogurt was $19.827 \pm 0.323 \mathrm{U} \mathrm{mL}^{-1}$, which was 63.01, 50.11, and 146.79\% higher than that of Bright Dairy Yogurt 1911, Junlebao and Nanjing Weigang, respectively. Fifty-four volatile flavor substances and 22,571 non-volatile flavor substances were found in yogurt. Compared to traditional fermented yogurt, 37 non-volatile metabolites in yogurt with millet enzymatic fermentation broth were significantly upregulated, including 2-phenyl ethanol, hesperidin, $\mathrm{N}$-acetylornithine and L-methionine, which were upregulated by 3169.6, 228.36, 271.22, and 55.67 times, respectively, thereby enriching the sensory and nutritional value of yogurt. Moreover, the manufacture of unpleasant volatile flavor substances was masked, making the product more compatible with consumers' tastes.

Keywords: lactic acid bacteria, superoxide dismutase, yogurt, HPLC-MS, HS-SPME-GC-MS

\section{INTRODUCTION}

The probiotic industry has embarked on a new windfall in the post-epidemic era, with market size of $\$ 51.2$ billion in 2020, and the global probiotic market is expected to grow at a CAGR of $6.9 \%$ from 2020 to 2025, while probiotic yogurt accounts for about $78 \%$ of the total global probiotic business $(1,2)$. Yogurt is a traditional dairy product manufactured by Lactobacillus delbrueckii subsp. bulgaricus and Streptococcus thermophilus by lactic acid fermentation (3). The direction of yogurt development is to develop high quality yogurt enriched with functional factors (4). The fermentation of Lactobacillus (LAB) manufactures superoxide dismutase (SOD) and peptides, which make yogurt richer in nutrients, easier to be digested and absorbed by the body (5).

SOD could scavenge superoxide anion free radicals and has various effects, such as anti-aging and anti-inflammatory. Therefore, SOD exhibited various applications in health care products, food and medicine (6). Many scholars have calculated the SOD content in microorganisms, and the results indicate that SOD is widespread in microorganisms. Therefore, the purpose of this study was to screened a strain of LAB with high SOD production to enhance the antioxidant function of yogurt. Some studies have found that using LAB with antioxidant activities can improve the antioxidant function of fermented products. After fermentation using 


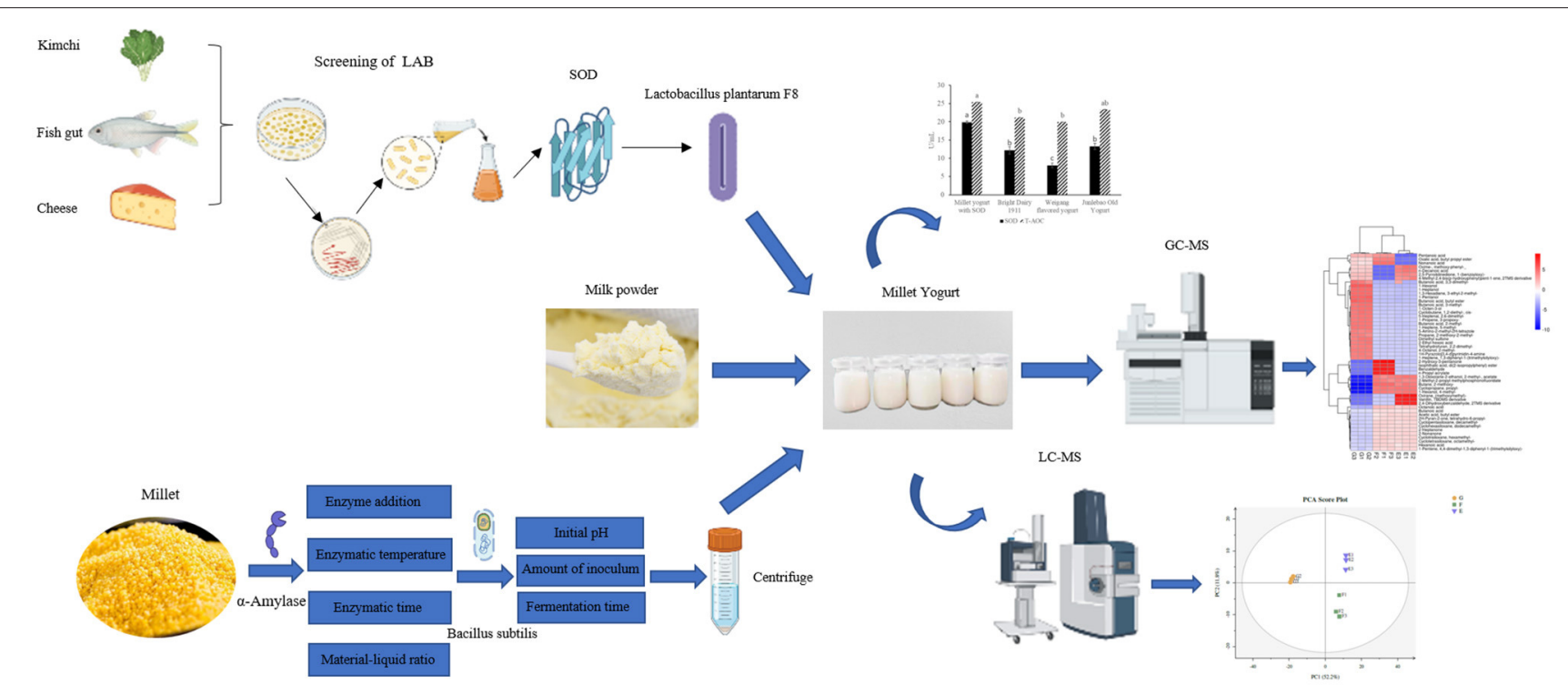

GRAPHICAL ABSTRACT | Novel millet-based flavored yogurt enriched with superoxide dismutase. Simplified experimental protocol for the preparation of SOD-enriched millet flavored yogurt.

the Lactiplantibacillus plantarum BCRC 10357, the total phenols and flavonoids in stone lotus increased, leading to improve the superoxide factor, DPPH free-radical scavenging ability, and the T-AOC of stone lotus (7). Soy milk fermented using Lacticaseibacillus rhamnosus CRLP81 has a higher T-AOC than the unfermented one, and it can inhibit DNA oxidative damage (8). Clinical studies have discovered that compared with the control group, the SOD activity in the serum of volunteers who ate kelp fermented with Lactobacillus sp. BJ20 is higher (9).

Fruit or plant ingredients are often added to yogurt to add sweetness and nutritional value, a practice that can also counteract the natural sourness of the yogurt. For example, pumpkin has been frequently used as a functional food for its antioxidant, antitumor, immunomodulatory, hypoglycemic and hepatoprotective properties (10). Millet contains some active antioxidant substances, such as minerals, vitamins and phenolic compounds (11). Adding millets to acid whey can be used to neutralize acid whey and enable efficient upcycling (12). Xiao Song reported the effect of polymerized whey protein and xanthan gum as thickeners of millet yogurt products on gel and microstructure qualities (13). However, the effect of millet enzymatic fermentation broth on the flavor and antioxidant capacity of yogurt was rarely reported. The treatment of millet was usually done through enzymatic digestion, but it has been improved by inoculating the millet with Bacillus subtilis after enzymatic digestion for further fermentation to enhance its utilization and nutritional value in this study. Bacillus subtilis is an essential biological leavening agent, many countries and regions use it to make traditional fermented legumes, such as Chinese tempeh and Japanese natto (14). Fermentation of millet enzymatic digests using Bacillus subtilis can improve its antioxidant capacity. According to reports, red bean products fermented using Bacillus subtilis IMR-NK1 exhibited significant antioxidant activity (15). The fermentation of black soybeans using Bacillus subtilis, which can improve the content of total phenols and isoflavones in the fermentation broth, can enhance its antioxidant activity (16). Therefore, we used Bacillus subtilis 109047 to ferment millet enzymatic solution, expecting to improve its antioxidant capacity as well as nutritional value.

However, yogurt will eventually be sold to the public, so the senses of yogurt are very crucial. The addition of high SODproducing LAB and millet fermentation might affect the flavor of yogurt, so it was necessary to detect the flavor. Most of the flavor compounds in yogurt are manufactured by milk fat lipolysis and the microbial conversion of lactose and citrate (17). More than 100 volatile flavors were found in yogurt, including carbonyl compounds, alcohols, acids, esters, hydrocarbons and aromatic compounds (3). HS-SPME-GC-MS and HPLC-MS can be used to determine volatile and non-volatile flavor substances in yogurt, respectively. Erkaya et al. have detected 34 volatile compounds using HS-SPME-GC-MS in yogurt made from the milk of sheep, cows, goats and buffaloes, of which acetaldehyde, diacetyl and acetylene were the major volatile compounds (18). A total of 196 non-volatile metabolites, including nucleosides, amino acids, carbohydrates and lipids, have been evaluated using UPLC-Q-TOF-MS (19).

This paper aimed to develop a millet-flavored yogurt enriched with SOD. Firstly, a LAB strain with high SOD production was screened, and its probiotic properties were investigated. Then, a millet enzyme fermentation broth was prepared and compounded with milk to develop SOD-rich millet yogurt. Finally, the effects of co-fermentation of screened strains with conventional fermenters on metabolites in yogurt and the effects of millet enzymatic fermentation broth on volatile and non-volatile flavor substances in yogurt were analyzed by metabolomics. 


\section{MATERIALS AND METHODS}

\section{Materials}

Kimchi, fish sausage and cheese, the ingredients used to screen for LAB, were purchased from the Nanjing Farmers' Market. Analysis reagents, including methanol, acetonitrile, methyl tertbutyl ether, formic acid and ammonium formate, were all purchased from Thermo. T-AOC kits and peptide assay kits were purchased from Solarbio.

\section{Methods}

\section{Screening of LAB With High SOD Production}

LAB was isolated from kimchi, fish sausage and cheese by spreading sample diluent on MRS medium that contained bromocresol purple indicator. The pyrogallol autotrophic method was used to determine the SOD enzyme activity of LAB. The strains were identified by $16 \mathrm{~S}$ rDNA sequencing (20).

\section{Probiotic Potential Evaluation of LAB}

The LAB was respectively inoculated into MRS with $\mathrm{pH}$ 2.5-8.5 and cultured for $24 \mathrm{~h}$ to observe the growth of the bacteria. The activated LAB was inoculated to MRS media with different $\mathrm{NaCl}$ content, with $\mathrm{NaCl}$ content of $1-8 \%(\mathrm{w} / \mathrm{v})$ and cultured for $16 \mathrm{~h}$ at $37^{\circ} \mathrm{C}$ to determine their anti-permeable pressure. The LAB was cultured in MRS at $\mathrm{pH} 7$ for $12 \mathrm{~h}$ to observe and evaluate the acid production capacity, and cultured in intestinal juice for $8 \mathrm{~h}$ and then in gastric juice for $3 \mathrm{~h}$ to calculate the survival rate (21). The $\mathrm{LAB}$ was successively passaged for 16 generations to determine the genetic stability, and the SOD production ability of the LAB was measured every two generations.

\section{Preparation and Optimization of Millet Enzymatic Hydrolysis Fermentation Broth}

The millet powder with a particle size of 80 mesh was prepared, mixed with water and heated at $80^{\circ} \mathrm{C}$ for $60 \mathrm{~min}$. The gelatinized millet powder was liquefied by alpha-amylase and then boiled. The supernatant was obtained and used to determine the TAOC and dextrose equivalent (DE) after the gelatinized millet powder had been centrifuged at $8000 \times \mathrm{g}$ for $10 \mathrm{~min}$ at $20^{\circ} \mathrm{C}$. The solid-liquid ratio (A), with enzyme amount (B), enzymatic digestion time (C) and enzymatic digestion temperature (D) were selected as four factors might affect the quality of millet enzymatic digestion solution for single-factor experiments.

\section{Changing Different Enzymolysis Conditions and the Optimizing Enzymolysis Scheme}

The millet enzymatic hydrolysate was fermented with Bacillus subtilis 109047 at $37^{\circ} \mathrm{C}$ for a specific period, and then the T-AOC was determined.

$$
\mathrm{T}-\operatorname{AOC}(\mathrm{U} / \mathrm{mL})=(\mathrm{x} \times \mathrm{V} 1) \div \mathrm{V} 2=34 \times \mathrm{x}
$$

Where V1 represents the total volume of the reaction $(0.204 \mathrm{~mL})$; V2 represents the volume of the sample in the reaction $(0.006 \mathrm{~mL})$, and $\mathrm{x}$ is calculated based on the standard curve.

$$
D E(\%)=(C \times V T) \div(m \times V S) \times 100
$$

Where C represents the amount of sugar (mg) based on the standard curve; VT represents the volume of the extract; $\mathrm{m}$ represents the mass of the sample, and VS represents the volume $(\mathrm{mL})$ of the sample used in the determination.

\section{Development of Millet Yogurt Rich in SOD}

The enzymatic fermentation broth of millet was centrifuged at $8,000 \times \mathrm{g}, 4^{\circ} \mathrm{C}$ for $10 \mathrm{~min}$. The supernatant was mixed with recovered milk and sterilized in a water bath at $95^{\circ} \mathrm{C}$ for $10 \mathrm{~min}$, then cooled in a water bath at $42^{\circ} \mathrm{C}$. Yogurt fermenter (Lactobacillus delbrueckii subsp. bulgaricus and Streptococcus thermophilus) with high SOD-producing LAB were inoculated into the above $42^{\circ} \mathrm{C}$ cooled recovered milk. The recovered milk was homogenized in a sterile environment and then fermented in an incubator at $42^{\circ} \mathrm{C}$ for $6 \mathrm{~h}(22)$. The yogurt with the most SOD content and best sensory were prepared by adding different amounts of starter, using different material-to-liquid ratios, adding different amounts of sucrose, adding phytochemicals and using different fermentation times.

\section{Sensory Evaluation}

The sensory evaluation was approved by the Ningbo University Institutional Review Board (NU-IRB). According to Mengdi Yin, sensory evaluation was performed using the quantitative descriptive analysis (QDA) method (23). The sensory evaluation was conducted by a professionally trained panel of 15 males and 15 females. The examined yogurt samples took into account four individual quality characteristics, including organization status, taste, odor and color. As shown in Table 1, the descriptors describing the characteristics of the examined samples and their definitions were decided by the panel after the training.

\section{Determination of SOD Content in Yogurt}

The $\mathrm{pH}$ of the yogurt was adjusted to 4.6 with $2 \mathrm{~mol} \mathrm{~L}^{-1} \mathrm{H}_{3} \mathrm{PO}_{4}$, and then the yogurt was bathed in water at $38^{\circ} \mathrm{C}$ for $5-10 \mathrm{~min}$. After the treated yogurt was centrifuged at $7,000 \times \mathrm{g}$ for $10 \mathrm{~min}$, the supernatant was removed and adjusted to $\mathrm{pH} 6.0$ with $0.5 \mathrm{~mol}$ $\mathrm{L}^{-1} \mathrm{NaOH}$ for subsequent SOD content test.

\section{Evaluation of Various Indicators of Yogurt}

The $\mathrm{pH}$ value of the final yogurt and the content of LAB was measured using a $\mathrm{pH}$ meter and the dilution coating method. The differences in T-AOC and peptide content among the final yogurt and Bright Dairy 1911, Junlebao and Nanjing Weigang flavor yogurt were compared.

\section{Analysis of Non-volatile Flavor Substances in Yogurt by HPLC-MS}

The yogurt was fermented in a $42^{\circ} \mathrm{C}$ incubator. The types of yogurts were as follows: traditional starter $(\mathrm{Y})$, traditional starter and Lactiplantibacillus plantarum subsp. plantarum F8 (Y-F8), and millet yogurt (Y-F8-M). Non-targeted metabolomics was used to analyze the effect of the addition of Lactiplantibacillus plantarum subsp. plantarum F8 and millet enzymatic fermentation broth on non-volatile flavor substances in yogurt (23-25). Metabolites were initially extracted from yogurt samples. Then, all samples were thawed at $4^{\circ} \mathrm{C}, 100 \mathrm{mg}$ 
TABLE 1 | Sensory rating scale.

\begin{tabular}{|c|c|c|}
\hline Items & Evaluation criteria & Score \\
\hline \multirow[t]{3}{*}{ Organizational status } & Yogurt has uniform texture, moderate viscosity and no whey precipitation. & $20-25$ \\
\hline & The yogurt has a relatively uniform texture, moderate viscosity and a small amount of whey precipitation. & $15-20$ \\
\hline & Yogurt has an uneven texture, low viscosity, and more whey precipitation. & $0-15$ \\
\hline \multirow[t]{3}{*}{ Taste } & The yogurt is moderately sweet and sour, delicate and smooth, and easily accepted. & $20-25$ \\
\hline & The yogurt is out of proportion to the sweet and sour, and the taste is a little rough but acceptable. & $15-20$ \\
\hline & Yogurt tastes too sour or too sweet and is not easy to accept. & $0-15$ \\
\hline \multirow[t]{3}{*}{ Odor } & It has the inherent whey flavor and pleasant odor of yogurt. & 20-25 \\
\hline & Yogurt has an inherent milky flavor that is relatively light and slightly sour. & $15-20$ \\
\hline & Yogurt has no creamy flavor, heavy sourness and incongruous odor. & $0-15$ \\
\hline \multirow[t]{3}{*}{ Color } & The yogurt is creamy white and shiny, uniform in color and free of impurities. & $20-25$ \\
\hline & The yogurt is creamy and slightly shiny, dark in color and free of impurities. & $15-20$ \\
\hline & Yogurt is milky white and lusterless, dull in color, with impurities. & $0-15$ \\
\hline
\end{tabular}

of which was transferred into $2 \mathrm{~mL}$ centrifuge tubes, added with $200 \mu \mathrm{L}$ of methanol and $200 \mu \mathrm{L}$ of MTBE into each centrifuge tube, and shaken for $60 \mathrm{~s}$. Afterward, the samples were centrifuged at $4^{\circ} \mathrm{C}$ for $10 \mathrm{~min}$ at $12,000 \mathrm{rpm}$, and the supernatant was filtered through a $0.22 \mu \mathrm{m}$ membrane to obtain the prepared samples for HPLC-MS (Thermo U300; $\mathrm{QE})$. The chromatographic conditions were as follows: a $1.8 \mu \mathrm{m}(2.1 \times 150 \mathrm{~mm})$ chromatographic column was used; injection port temperature of $8^{\circ} \mathrm{C}$; flow rate of $0.25 \mathrm{~mL} \mathrm{~min}^{-1}$; column temperature of $40^{\circ} \mathrm{C}$; and a $2 \mu \mathrm{L}$ injection was used for gradient elution. The mobile phase was positive ion $0.1 \%$ formic acid water $0.1 \%$ formic acid acetonitrile and negative ion $5 \mathrm{mM}$ ammonium formate water acetonitrile. Finally, a mass spectrometer was used an electrospray ionization source in positive and negative ionization mode with positive and negative ion spray voltages of 3.50 and $2.50 \mathrm{kV}$, respectively, a sheath gas of $30 \mathrm{arb}$, and an auxiliary gas of $10 \mathrm{arb}$. The capillary was set at $325^{\circ} \mathrm{C}$, and a full scan was performed with a resolution of 70,000, a scan range of $81-1,000$, and a secondary cleavage with $\mathrm{HCD}$, with a collision voltage of $30 \mathrm{eV}(19,26,27)$.

\section{Analysis of Volatile Flavor Compounds in Yogurt by HS-SPME-GC-MS}

The HS-SPME device was used to extract volatile flavor compounds in yogurt for $60 \mathrm{~min}$ at $50^{\circ} \mathrm{C}$ and $350 \mathrm{rpm}$. The GC-MS instrument make and model was Agilent 8890 GC System $+5977 \mathrm{~B} / \mathrm{MSD}$. The chromatographic conditions were set at an initial temperature of $35^{\circ} \mathrm{C}$ for $3 \mathrm{~min}, 4^{\circ} \mathrm{C} \mathrm{min}^{-1}$ to $140^{\circ} \mathrm{C}$ for $1 \mathrm{~min}$, and $10^{\circ} \mathrm{C} \mathrm{m^{-1 }}$ to $250^{\circ} \mathrm{C}$ for $3 \mathrm{~min}$. The injection temperature was set at $250^{\circ} \mathrm{C}$; the carrier gas was $\mathrm{He}$, and the flow rate was $1.0 \mathrm{~mL} \mathrm{~min}^{-1}$. The mass spectrometry conditions were as follows: ionization mode $\mathrm{EI}$ of $70 \mathrm{eV}$, ion source temperature of $230^{\circ} \mathrm{C}$, mass scanning range of 33-650 AMV, and emission voltage of $100 \mu \mathrm{A}(22,28)$. Agilent MssHunter Qualitative Analysis B.07.00 software and NIST14.LMS database provided by Agilent were used for data comparison, while we referred to metabolomics analysis methods from other scholars (29-32).
TABLE 2 | SOD content of lactic acid bacteria screened.

\begin{tabular}{lccc}
\hline Bacteria number & SOD (U/g) & Bacteria number & SOD (U/g) \\
\hline F1 & $597.48 \pm 2.74$ & F12 & $2143.97 \pm 0.68$ \\
F2 & $363.07 \pm 3.68$ & F13 & $854.29 \pm 6.71$ \\
F3 & $731.47 \pm 2.18$ & F14 & $1238.99 \pm 9.56$ \\
F4 & $682.85 \pm 2.25$ & F15 & $871.56 \pm 2.32$ \\
F5 & $1379.85 \pm 1.93$ & F16 & $1358.69 \pm 7.21$ \\
F6 & $659.46 \pm 3.37$ & F17 & $587.11 \pm 5.79$ \\
F7 & $489.44 \pm 3.09$ & $F 18$ & $607.05 \pm 7.91$ \\
F8 & $2476.21 \pm 1.52$ & $F 19$ & $563.78 \pm 4.23$ \\
F9 & $487.54 \pm 4.33$ & F20 & $454.26 \pm 3.56$ \\
F10 & $963.44 \pm 4.37$ & $F 21$ & $284.55 \pm 5.44$ \\
F11 & $1594.24 \pm 5.05$ & & \\
\hline
\end{tabular}

SOD, Superoxide dismutase.

\section{Statistical Analysis}

Differences between samples were analyzed using one-way analysis of variance (ANOVA) and Duncan's multiple range tests using SPSS. A $P$-value of $<0.05$ was considered to be statistically significant. All experiments were conducted three times in parallel. Bioinformatics analyses such as PCA plots and heat maps were performed using the OmicStudio tool at https:// www.omicstudio.cn/tool.

\section{RESULTS AND DISCUSSION Screening of LAB With High SOD Production}

Twenty-one strains of LAB were screened and their SOD enzyme activities were determined. The results were indicated in Table 2, a target strain with high SOD production was screened, and the SOD content of this strain reached $2476.21 \pm 1.52 \mathrm{U} \mathrm{g}^{-1}$. This strain had the highest SOD content among the 21 strains of LAB. Based on the $16 \mathrm{~S}$ rDNA sequence, the screened F8 strain was identified as: Lactiplantibacillus plantarum subsp. plantarum (Figure 1). 
A

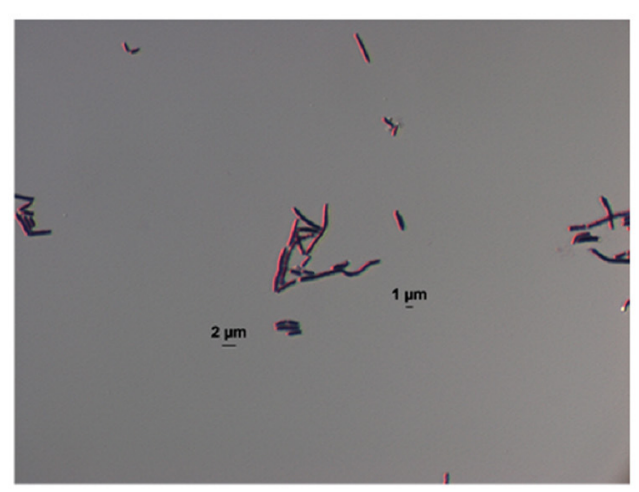

B

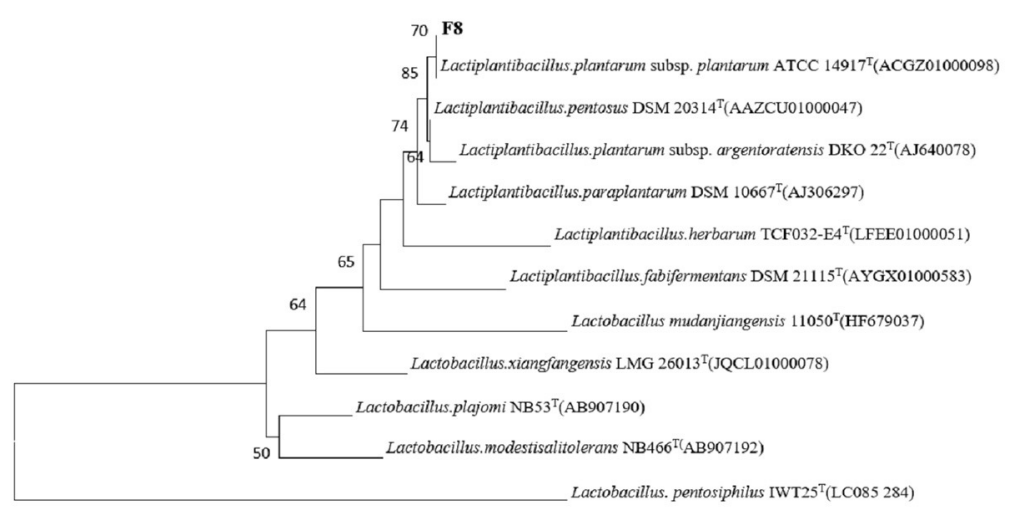

0.005

FIGURE 1 | (A) Results of Gram staining of Lactiplantibacillus plantarum subsp. plantarum F8. (B) Phylogenetic tree of 16S rDNA sequence of the target strain Lactiplantibacillus plantarum subsp. plantarum F8 and related species.

As indicated in Figure 2A, the LAB grew well at a $\mathrm{pH}$ of 4 , and the LAB needed good acid resistance to maintain their activity in a gastric acid environment (33). Figure $\mathbf{2 B}$ shows that the bacteria could decrease the $\mathrm{pH}$ of the fermentation broth to 4.38 within $12 \mathrm{~h}$, which indicated the high acid production capacity of this LAB. This was close to the results of $\mathrm{A} \mathrm{H} \mathrm{X}$ G screening LAB for fermentation of high-quality yogurt (34). The strain could withstand the osmotic pressure of $8.0 \%$ sodium chloride, indicating that the strain might also have application prospects in fermented kimchi and other low-salt pickled foods. The prerequisite that $\mathrm{LAB}$ could play a beneficial role in the intestine is to be able to tolerate the gastrointestinal environment and survive (31). As shown in Figure 2C, the bacterium exhibited very good tolerance in gastrointestinal fluids, reaching $58.54 \%$ survival in intestinal fluids for $8 \mathrm{~h}$ and $61.7 \%$ survival in gastric fluids for $2 \mathrm{~h}$. This is similar to the result of $\mathrm{Wu}$ et al. (35). In addition, the bacterium exhibited excellent genetic stability of SOD (Figure 2D), which is similar to Chooruk A's results (36).

\section{Preparation and Optimization of Millet Enzymatic Hydrolysis Fermentation Broth}

In the single-factor experiment of millet enzymatic digestion (Figures 3A-D), the best enzymatic digestion conditions were as follows: solid-liquid $\operatorname{ratio}(\mathrm{A})$ of 1:10 (w/v), amylase addition (B) of $16 \mathrm{U} \mathrm{g}^{-1}$, enzymatic digestion time (C) of $70 \mathrm{~min}$ and enzymatic digestion temperature (D) of $65^{\circ} \mathrm{C}$. Based on the orthogonal test results (Table 3 ), the order of factors affecting the $\mathrm{DE}$ value of millet enzymatic hydrolysate was $\mathrm{D}>\mathrm{B}>\mathrm{C}>\mathrm{A}$. When the T-AOC was used as the standard, the order of factors affecting the T-AOC of millet enzymatic hydrolysate was $\mathrm{A}>\mathrm{C}>\mathrm{B}>\mathrm{D}$. The best combination was obtained from $A_{1} B_{2} C_{1} D_{3}$. Considering these two factors, the best combination was determined as $A_{1} B_{1} C_{3} D_{1}$, which is, the ratio of the material to liquid was $1: 10(\mathrm{w} / \mathrm{v})$. The amount of enzyme added was $16 \mathrm{U}$ $\mathrm{g}^{-1}$, the enzymatic hydrolysis time was $80 \mathrm{~min}$ and the enzymatic hydrolysis temperature was $65^{\circ} \mathrm{C}$. Three repetitive experiments were conducted under this condition. The average $\mathrm{DE}$ value was $40.03 \pm 0.85 \%$ and the T-AOC was $26.24 \pm 0.53 \mathrm{U} \mathrm{mL}^{-1}$. The DE value increased by $7.35 \%$ and the T-AOC increased by $19.71 \%$. This result was consistent with previous reports that the increased antioxidant capacity of millet enzymatic hydrolysis might be mainly related to the contain of polyphenols and vitamins as well as SOD contained in millet (11).

As indicated in Figure 3E, with the extension of fermentation time, the T-AOC of millet enzymatic hydrolysis fermentation broth initially increased and then reduced, reaching the maximum at $36 \mathrm{~h}$. The T-AOC of the fermentation broth continued to rise with the increase in the inoculation amount. When the inoculum amount was $3 \%$, the T-AOC of the fermentation broth was the largest. With the increase of $\mathrm{pH}$, the T-AOC increased and then decreased, reaching a maximum value of $44.53 \pm 1.08 \mathrm{U} \mathrm{mL}^{-1}$ at $\mathrm{pH} 7$. As the fermentation time was prolonged, the content of Bacillus subtilis secreted protease, and other enzymes increased gradually. Large proteins were hydrolyzed into peptides and anti-oxidant substances such as flavonoids and polyphenols, which were found in the millet enzymatic hydrolysate (37). Therefore, it might be that the increase in these antioxidants was what made the T-AOC increased. Afterward, some active peptides were continuously hydrolyzed into free amino acids (38). As time increased, flavonoids and polyphenols continued to decrease, and the TAOC declined. The amount of inoculation greatly influenced the primary and secondary metabolism of microorganisms (34). When the inoculate volume was at a low level, the enzyme and peptide content in the fermentation broth increased as inoculate volume increases, causing an increase in T-AOC. If the bacteria were cultured in high density, the microorganism's growth and reproduction will be inhibited. Therefore, the enzyme manufactured was relatively small, and the T-AOC was reduced. Therefore, the optimal fermentation conditions were as follows: fermentation time of $36 \mathrm{~h}$, Bacillus subtilis addition amount of $3 \%$, and initial $\mathrm{pH}$ of 7 . Compared with 
A

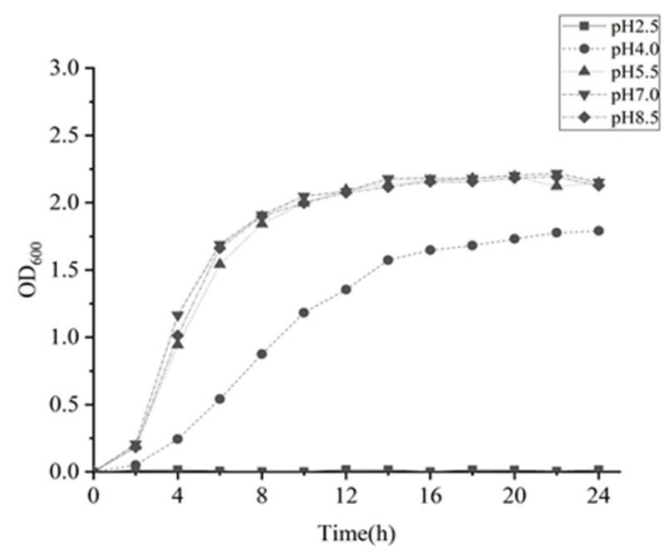

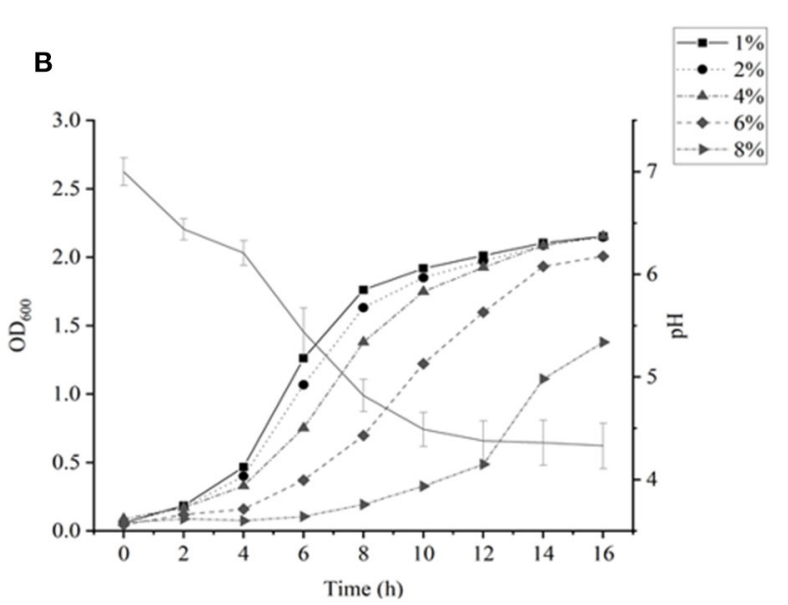

Gastric juice -Intestinal juice c

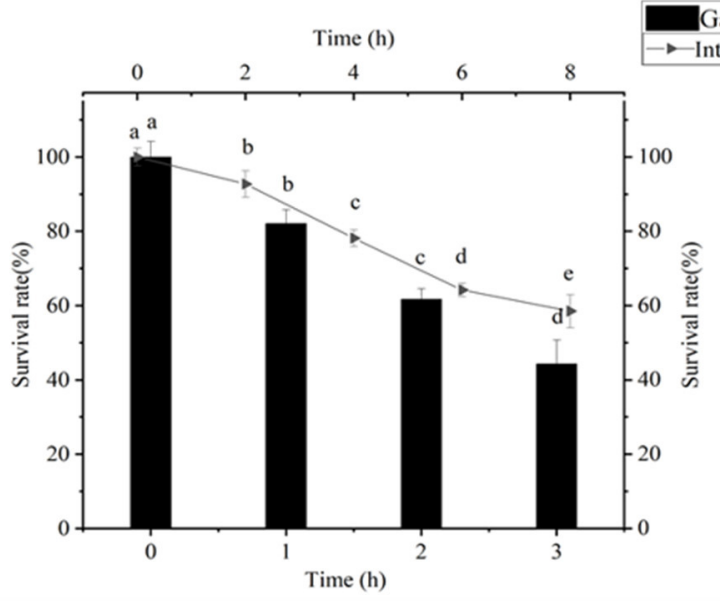

\section{D}

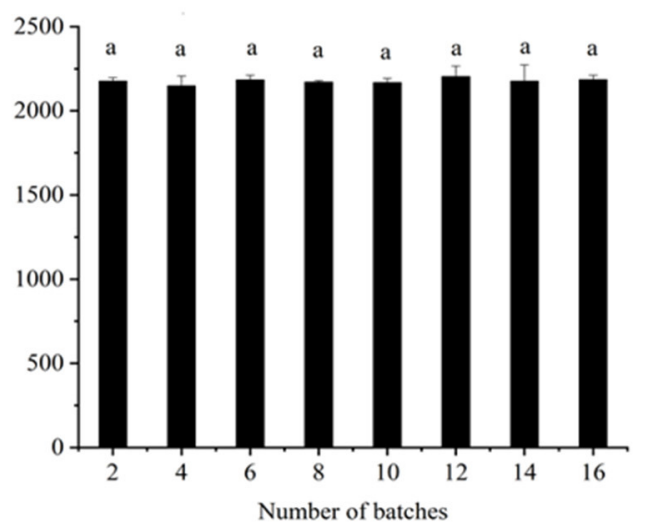

FIGURE 2 | (A) Result of the acid resistance test of Lactiplantibacillus plantarum subsp. plantarum F8. (B) Determination of acid production capacity; the pH of the medium reduced from 7 to 4.38 within $12 \mathrm{~h} ; 1-8 \% \mathrm{NaCl}$ was added to the MRS medium, and the growth of the bacteria was measured in $2-16 \mathrm{~h}$. (C) Survival rate of $\mathrm{LAB}$ in artificial intestinal juice within $8 \mathrm{~h}$. Survival rate of LAB in artificial intestinal juice for $3 \mathrm{~h}$. (D) LAB were successively passaged for $16 \mathrm{generations,} \mathrm{and} \mathrm{the} \mathrm{SOD}$ content was measured every two generations. The genetic performance was stable. Significant differences were indicated by the letters a, $b$ and $c$. Any difference with the same letter was considered not significant $(p>0.05)$ and any difference with a different letter was considered significant $(p<0.05)$.

initial fermentation, the T-AOC of the fermentation broth has increased by $69.7 \%$. Bacillus subtilis might improve the nutritional value and antioxidant capacity of millet enzymes by producing nattokinase, SOD, and catalase (37).

\section{Development of Millet Yogurt Rich in SOD}

During the fermentation of yogurt, about $20 \%$ of sugar and protein was broken down into small molecules and fatty acids, which improved the utilization of nutrients. In addition to retaining all the nutrients of yogurt, lactic acid, amino acids and fatty acids produced by LAB were easily absorbed by the body (39). The SOD content in yogurt reached the maximum at 2:8 $(\mathrm{v} / \mathrm{v})$ (Figure 4A), and at this time the yogurt had moderate viscosity, no whey precipitation, special yogurt taste, creamy white color and glossy. The SOD content in yogurt reached the maximum at 2:8 (v/v), and at this time the yogurt had moderate viscosity, no whey precipitation, special yogurt taste, creamy white color and glossy. The addition of sucrose had almost no effect on the SOD content in yogurt, but had a great influence on the sensory evaluation score of yogurt taste (Figure 4B), which is consistent with previous reports (6).

The SOD content increased significantly with increasing inoculum, reaching a maximum at $4 \%(\mathrm{v} / \mathrm{v})$ (Figure 4C), indicating that the addition of LAB with high SOD production might affect the SOD content of yogurt. This was similar to the results of Wang et al. who fermented stone lotus with Lactiplantibacillus plantarum BCRC 10357 (7). As shown in Figure 4D, the SOD content gradually increased with the increase of yogurt fermenters, but there was no significant difference. This might be due to the fact that for yogurt fermenters were composed of Lactobacillus delbrueckii subsp. bulgaricus and Streptococcus thermophilus, which could also produce small amounts of SOD during fermentation (22). The SOD content in yogurt reached a maximum at $8 \mathrm{~h}$ (Figure $4 \mathrm{E}$ ), 


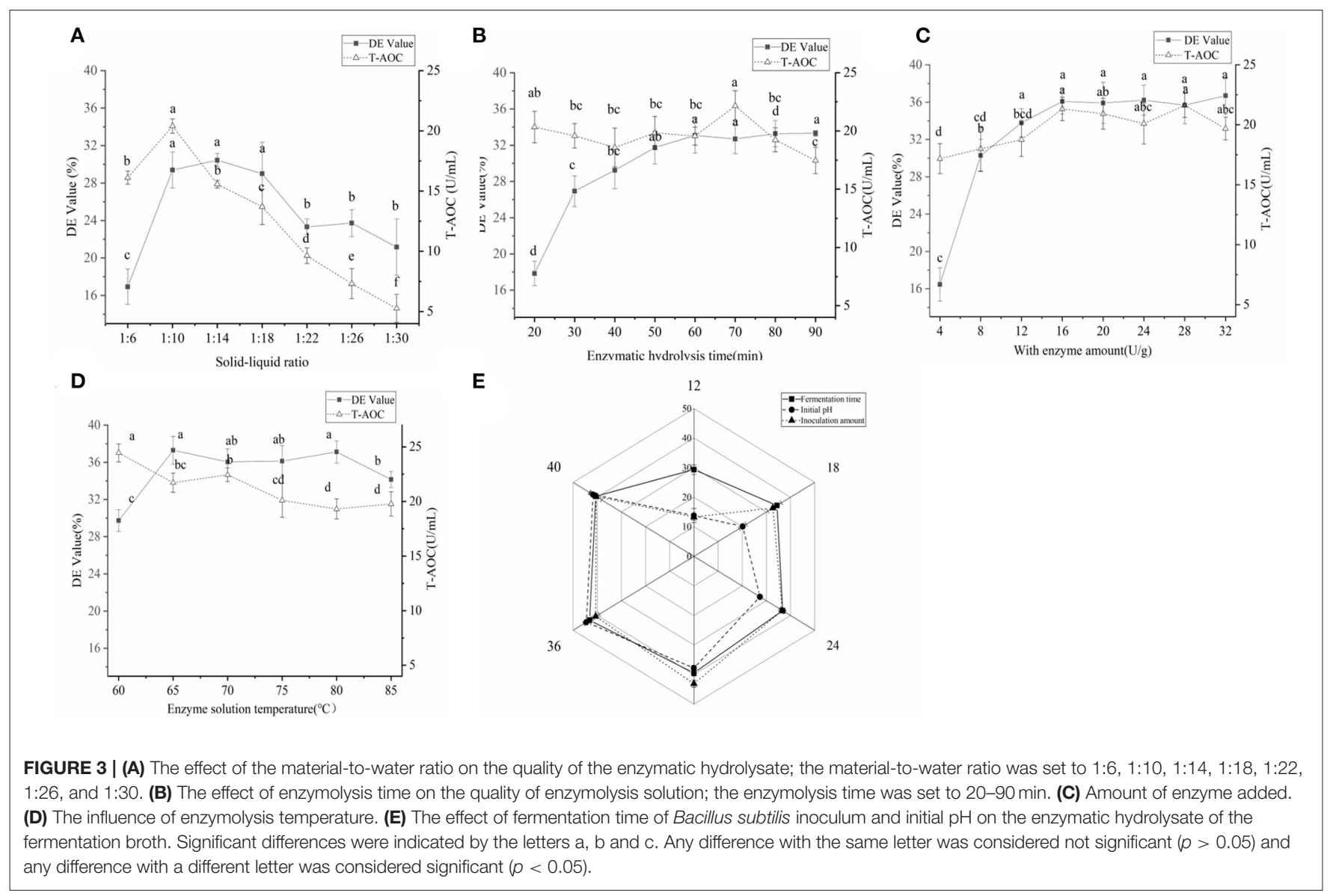

TABLE 3 | Analysis table of orthogonal test results.

\begin{tabular}{|c|c|c|c|c|c|c|c|c|}
\hline \multirow[b]{2}{*}{$\mathrm{K}_{1}$} & \multicolumn{4}{|c|}{ Take DE value as standard (\%) } & \multicolumn{4}{|c|}{ Take T-AOC as standard (U/mL) } \\
\hline & 110.81 & 112.41 & 108.27 & 114.39 & 77.03 & 71.33 & 72.59 & 70.48 \\
\hline $\mathrm{K}_{2}$ & 109.86 & 112.20 & 108.87 & 100.92 & 73.61 & 72.24 & 70.09 & 71.26 \\
\hline $\mathrm{K}_{2}$ & 108.48 & 104.61 & 112.08 & 113.91 & 62.73 & 69.80 & 70.69 & 71.63 \\
\hline $\mathrm{k}_{1}$ & 36.94 & 37.47 & 36.09 & 38.13 & 25.68 & 23.78 & 24.20 & 23.49 \\
\hline $\mathrm{k}_{2}$ & 36.62 & 37.40 & 36.29 & 33.64 & 24.54 & 24.08 & 23.36 & 23.75 \\
\hline Excellent level & $A_{1}$ & $B_{1}$ & $\mathrm{C}_{3}$ & $D_{1}$ & $A_{1}$ & $\mathrm{~B}_{2}$ & $C_{1}$ & $D_{3}$ \\
\hline
\end{tabular}

$D E$, Dextrose Equivalent; T-AOC, total antioxidant capacity.

after which it gradually decreased. This could be explained by the gradual decrease in $\mathrm{pH}$ with increasing fermentation time, which might affect the SOD production and SOD enzyme activity of $\operatorname{LAB}(6)$.

To obtain the best recipe for making millet yogurt, an orthogonal test was conducted. Based on the orthogonal results (Table 4), the order of influence on the SOD content in millet yogurt was $\mathrm{A}>\mathrm{B}>\mathrm{D}>\mathrm{C}$. The optimal conditions were as follows: a liquid-to-milk ratio of 2:8 (v/v), amount of added Lactiplantibacillus plantarum subsp. plantarum F8 of 3\% (v/v), amount of added direct starter of $0.25 \%(\mathrm{w} / \mathrm{v})$, and fermentation time of $6 \mathrm{~h}$. Re-experiments verified that the SOD content in yogurt was $19.827 \pm 0.323 \mathrm{U} \mathrm{mL}^{-1}$, which was increased by $7.25 \%$ compared with the previous orthogonal optimization. Feng et al. determined the SOD content in human and certain livestock milk and found that sow milk, dog milk, human milk and cow milk were $69.6,34.9,7.1$, and $3.2 \mathrm{U} \mathrm{mL}^{-1}$, respectively (40). By contrast, the SOD content of the millet yogurt developed in this experiment was similar to that of dog milk, and it was much higher than that of human milk and cow milk. SOD is the only enzyme known to directly scavenge free radicals, breaking down superoxide dismutase in organisms into oxygen 

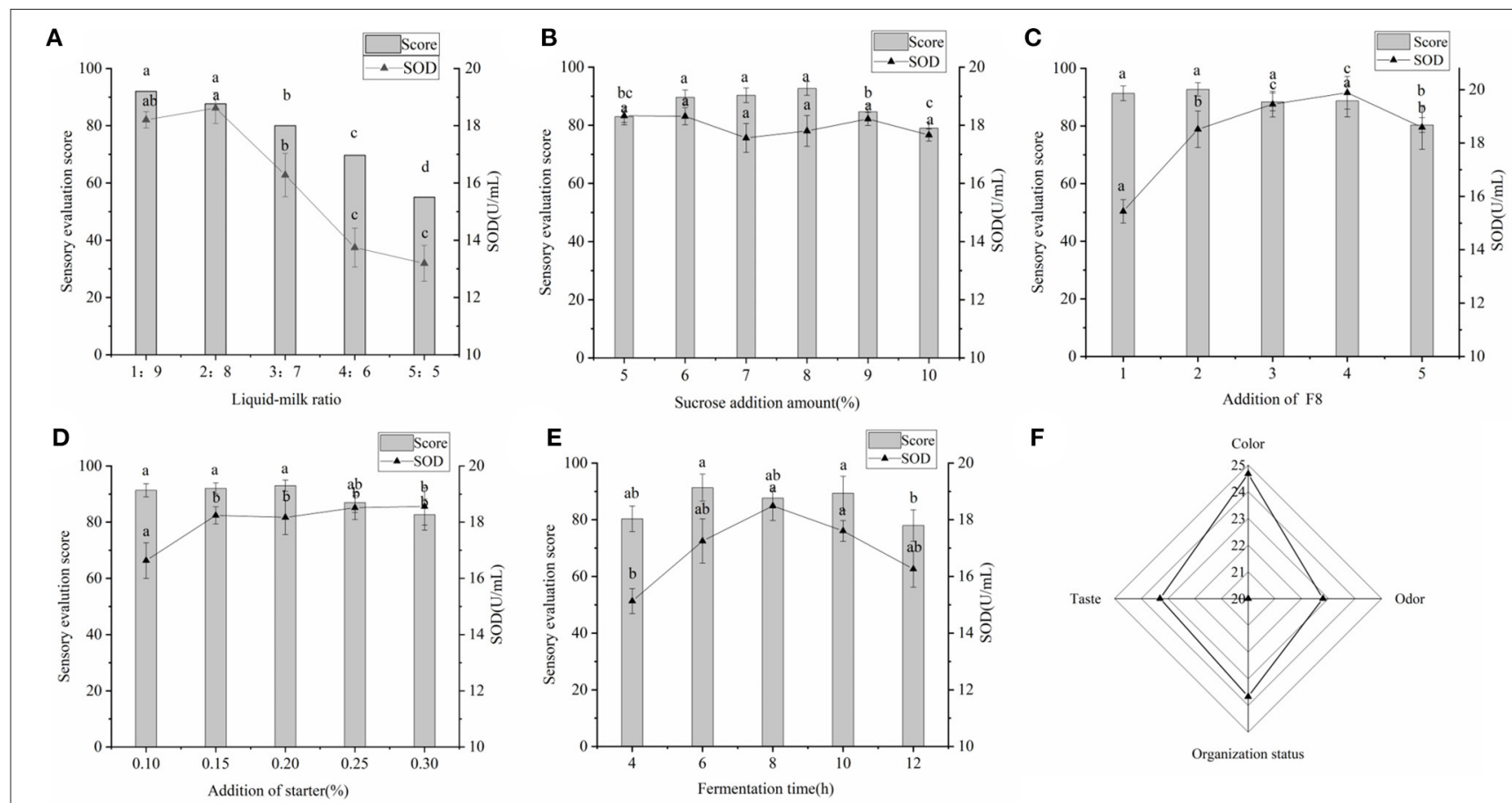

FIGURE 4 | (A) Effects of raw material ratio on SOD content and sensory. The ratio of millet enzymatic fermentation broth and fresh milk was set to 1:9, 2:8, 3:7, 4:6, and 5:5. (B) Effects of added sucrose on SOD content and sensory. The amount of sucrose added was set to 5-10\%. (C) Effects of the amount of Lactiplantibacillus plantarum subsp. plantarum F8 on SOD content and sensory sense. (D) The effect of direct-input starter on the yogurt quality. (E) The influence of fermentation time on yogurt. (F) Sensory evaluation chart of final yogurt. Significant differences were indicated by the letters $a, b$ and $c$. Any difference with the same letter was considered not significant $(p>0.05)$ and any difference with a different letter was considered significant $(p<0.05)$.

and hydrogen peroxide with high specificity and efficiency (6). Overexpression of SOD has also been shown to mitigate radiation-induced damage (41). The SOD-rich millet yogurt we have developed was expected to appear in the recipes of people with pre-cancer or to prevent the development of diseases such as cancer.

Figure $4 \mathrm{~F}$ was drawn from the results of three independent yogurt sensory evaluations. The results indicated that the yogurt was of high quality. The final yogurt was creamy white, glossy, with a pleasant milk flavor, uniform texture and moderate sweetness. The color, odor, texture and taste were 24.7, 22.8, 23.7, and 23.3 points, respectively. The results were similar to those of Yin $\mathrm{M}$ et al. for the sensory evaluation of yogurt (23). The $\mathrm{pH}$ of the final yogurt ranged from 4.1 to 4.3 , the amount of LAB in fermented millet yogurt was $1.43 \times 10^{8} \mathrm{CFU} \mathrm{mL} \mathrm{m}^{-1}$ $>1 \times 10^{6} \mathrm{CFU} \mathrm{mL} \mathrm{m}^{-1}$, and no contamination of miscellaneous bacteria was detected in the yogurt. These were in accordance with the regulations for fermented milk (42). The SOD-rich millet yogurt developed by this project was compared with three types of yogurts available on the market. The results were shown in Table 5. The SOD content of the final yogurt was 63.01, 50.11, and $146.79 \%$ higher than the ordinary yogurt available on the market, including Bright Dairy 1911, Junlebao Old Yogurt and Weigang-flavored yogurt. This might be due to the high SODproduction LAB we used, in addition, the SOD contained in the millet enzymatic hydrolysis fermentation broth also promoted the total SOD in yogurt (6). The T-AOC of the final yogurt was $19.86,8.67$, and $27.24 \%$ higher than the aforementioned ordinary yogurt, respectively. This might be because millet yogurt contained antioxidant substances such as polyphenols, flavonoids and vitamin E from millet, and antioxidant enzymes such as SOD and catalase from Bacillus subtilis (37). The peptide content was slightly higher than Weigang-flavored yogurt and lower than Bright Dairy 1911 and Junlebao Old Yogurt. These results indicated that yogurt co-fermented with millet enzymatic digest has high-quality properties and significant antioxidant potential, and thus has great potential as a new functional food in the food industry.

\section{Analysis of Non-volatile Flavor Substances in Yogurt by HPLC-MS}

Twenty-two thousand five hundred and seventy-one non-volatile flavor substances from traditional starter $(\mathrm{Y})$, traditional starter and Lactiplantibacillus plantarum subsp. plantarum F8 (YF8), millet yogurt (Y-F8-M) were screened by LC-MS, and the relevant differences among metabolites were determined. The screening conditions were $p$-value $\leq 0.05$, VIP $\geq 1$, and Fold_Change $\geq 1.5$ or $\leq 0.667$. Two hundred differential metabolites were selected (43). Multivariate statistical analysis was conducted to evaluate non-volatile metabolites and identify any potential variability associated with specific conditions. The PCA score was calculated to determine whether the sample 
TABLE 4 | $\mathrm{L}_{9}\left(4^{3}\right)$ Orthogonal results of millet yogurt rich in SOD.

\begin{tabular}{|c|c|c|c|c|c|}
\hline \multirow[t]{2}{*}{ Level } & \multicolumn{4}{|c|}{ Experimental factors } & \multirow{2}{*}{$\frac{\text { Results }}{\text { SOD (U/mL) }}$} \\
\hline & A & B & C & D & \\
\hline 1 & 1 & 1 & 1 & 1 & $16.720 \pm 0.200$ \\
\hline 2 & 1 & 2 & 2 & 2 & $17.515 \pm 0.745$ \\
\hline 3 & 1 & 3 & 3 & 3 & $18.621 \pm 0.227$ \\
\hline 4 & 2 & 1 & 2 & 3 & $17.523 \pm 0.175$ \\
\hline 5 & 2 & 2 & 3 & 1 & $19.827 \pm 0.323$ \\
\hline 6 & 2 & 3 & 1 & 2 & $17.634 \pm 0.540$ \\
\hline 7 & 3 & 1 & 3 & 2 & $15.317 \pm 1.326$ \\
\hline 8 & 3 & 2 & 1 & 3 & $16.486 \pm 0.361$ \\
\hline 9 & 3 & 3 & 2 & 1 & $17.012 \pm 0.027$ \\
\hline $\mathrm{K}_{1}$ & 53.856 & 49.560 & 50.840 & 53.559 & / \\
\hline $\mathrm{K}_{2}$ & 54.984 & 53.828 & 52.050 & 50.466 & / \\
\hline $\mathrm{K}_{3}$ & 48.815 & 53.267 & 53.765 & 52.630 & / \\
\hline $\mathrm{k}_{1}$ & 17.610 & 16.520 & 16.946 & 17.850 & / \\
\hline $\mathrm{k}_{2}$ & 18.328 & 17.942 & 17.350 & 16.822 & / \\
\hline $\mathrm{k}_{3}$ & 16.271 & 17.755 & 17.921 & 17.540 & / \\
\hline$R$ & 2.057 & 1.422 & 0.975 & 1.031 & / \\
\hline Excellent level & $\mathrm{A}_{2}$ & $\mathrm{~B}_{2}$ & $\mathrm{C}_{3}$ & $\mathrm{D}_{1}$ & / \\
\hline
\end{tabular}

SOD, Superoxide dismutase.

TABLE 5 | Comparison of the contents of various substances in ordinary yogurt.

\begin{tabular}{lccc}
\hline Category & SOD $(\mathbf{U} / \mathbf{m L})$ & T-AOC $(\mathbf{U} / \mathbf{m L})$ & Peptide $(\mathbf{m g} / \mathbf{m L})$ \\
\hline Millet yogurt with SOD & $19.827 \pm 0.323^{\mathrm{a}}$ & $25.331 \pm 1.691^{\mathrm{a}}$ & $1.282 \pm 0.049^{\mathrm{c}}$ \\
Bright Dairy 1911 & $12.163 \pm 1.001^{\mathrm{b}}$ & $21.134 \pm 1.591^{\mathrm{b}}$ & $1.649 \pm 0.020^{\mathrm{b}}$ \\
Weigang flavored yogurt & $8.034 \pm 0.614^{\mathrm{C}}$ & $19.908 \pm 2.593^{\mathrm{b}}$ & $1.057 \pm 0.068^{\mathrm{d}}$ \\
Junlebao Old Yogurt & $13.208 \pm 0.892^{\mathrm{b}}$ & $23.311 \pm 1.207^{\mathrm{ab}}$ & $1.881 \pm 0.047^{\mathrm{a}}$
\end{tabular}

$S O D$, Superoxide dismutase; T-AOC, total antioxidant capacity. Any difference with the same letter was considered not significant $(p>0.05)$ and any difference with a different letter was considered significant $(p<0.05)$.

repeatability in the group was sufficiently large (44). As shown in Figure 5, the repeatability of the three yogurt samples was better. The distance of the three yogurt samples was relatively close, the difference differed. The three yogurt samples were separated from the PC1 (52.2\%), and along the PC2 direction (11.8\%).

A Heat-map was drawn to distinguish the three groups of sample metabolites (Figure 6). Each small square indicated the metabolic substance content, and the colors indicated the metabolite content. A total of 22,571 non-volatile flavor substances were found in yogurt. Compared with Group E, the change of non-volatile flavor substances in Group $\mathrm{F}$ might be mainly related to the 9 metabolic pathways with high significance and high pathway impact in Lactiplantibacillus plantarum subsp. plantarum F8. Including 2-Oxocarboxylic acid metabolism, Aminobenzoate degradation, Biosynthesis of alkaloids derived from ornithine, lysine and nicotinic acid, Degradation of aromatic compounds, $\mathrm{ABC}$ transporters, Biosynthesis of amino acids, Microbial metabolism in diverse environments and

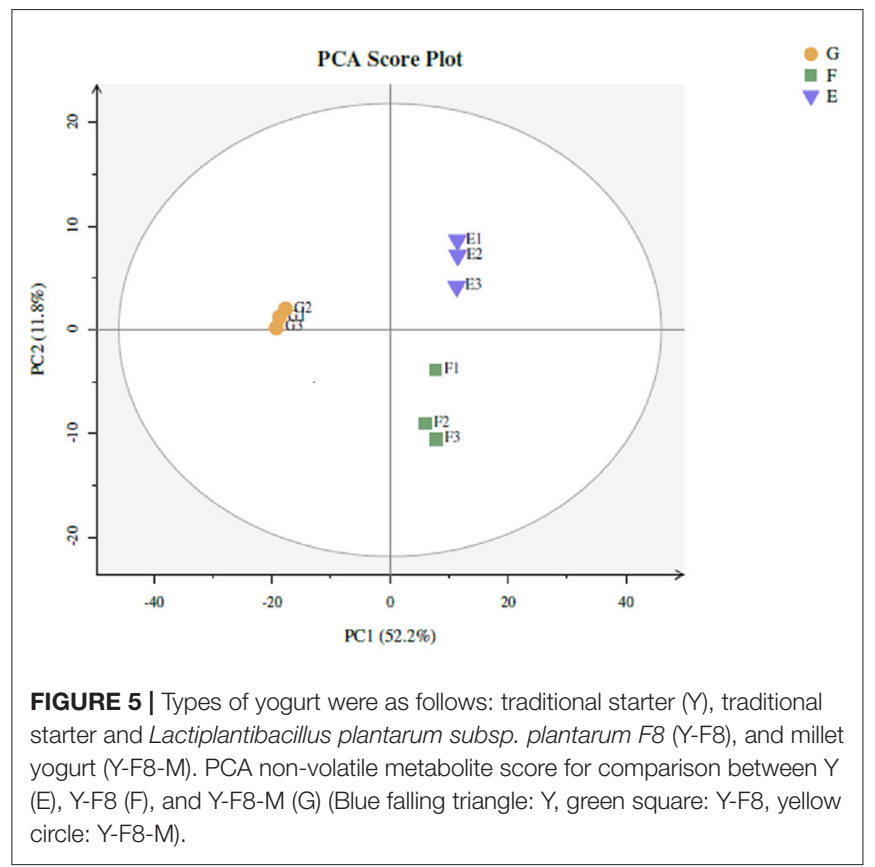

Biosynthesis of secondary metabolites, as shown in Figure 7A. Compared with Group E, 12 metabolites were found to be significant upregulated in group F. Among them, the content of 4-Hydroxy-3-methoxybenzenemethanol was increased by 6.48 times. It had milk, cream and coconut aroma, which was a common baked food natural spice (3). The L-Threonine content was increased 5.45 times, which was considered an essential 


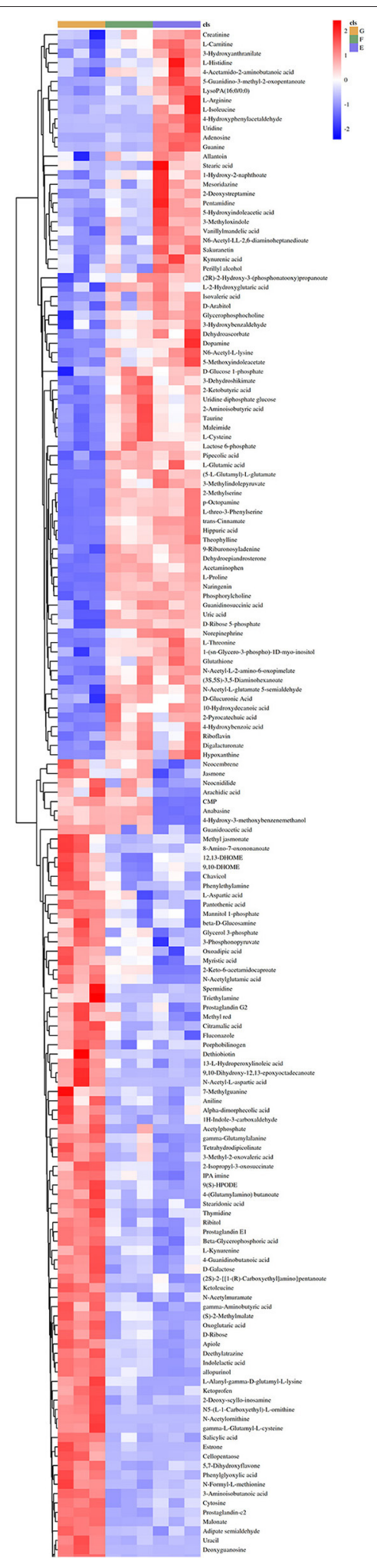

FIGURE 6 | Heat-map and hierarchical clustering of non-volatile metabolite profiles in yogurt. The dendrogram represents sample clusters based on Pearson's correlation coefficient with average linkage. The normalized non-volatile metabolite abundance was visualized by color: red indicates the highest values, and blue indicates the lowest values (E: Y, F: Y-F8, G: Y-F8-M). amino acid with a slightly sweet taste, and it was produced by the action of threonine synthase with high serine phosphate as a substrate (17). The content of allopurinol increased by 4.57 times, which is an isomer of xanthine. It could reduce the production of oxygen free radicals in ischemic re-injection damage to achieve antioxidant effects, so it was often used in primary and secondary hyperuricemia (45). This might also be partly responsible for the high antioxidant capacity of millet yogurt. Arachidonic acid was increased by 2.01 times, and it was a polyunsaturated fatty acid that might play a role in reducing the prevention of diabetes and tumors. In Lactiplantibacillus plantarum subsp. plantarum, it was produced by phosphatidylcholine and $\mathrm{H}_{2} \mathrm{O}$ through the arachidonic acid metabolic pathway catalyzed by phospholipase A (46). In addition, nine metabolites were downregulated, with uridine, adenosine and guanine decreasing by $28.57,4.14$, and 4.34 times, respectively. Adenosine is an important intermediate used in the synthesis of adenine, and an increase in purine levels in the body leads to an increase in uric acid, which ultimately leads to hyperuricemia in humans. This was consistent with the reported ability of LAB to absorb and metabolize exogenous nucleosides and purine bases (47). Furthermore, this Lactiplantibacillus plantarum subsp. plantarum has a strong nucleoside catabolic capacity and might be applied in the diet of patients with hyperuricemia. The metabolomic analysis indicated that Lactiplantibacillus plantarum subsp. plantarum might have a strong probiotic potential and application value.

Compared with Group E, the production of differential nonvolatile flavor substances in Group $G$ was related not only to millet enzymatic hydrolysis fermentation broth, but also related to the 13 metabolic pathways with high significance and high pathway impact in Lactiplantibacillus plantarum subsp. Plantarum F8. Including Bile secretion, Biosynthesis of alkaloids derived from shikimate pathway, Arginine and proline metabolism, Carbon metabolism, Biosynthesis of alkaloids derived from ornithine, lysine and nicotinic acid, AminoacyltRNA biosynthesis, Central carbon metabolism in cancer, 2Oxocarboxylic acid metabolism, ABC transporters, Biosynthesis of cofactors, Biosynthesis of plant secondary metabolites, Biosynthesis of amino acids and Biosynthesis of secondary metabolites, as shown in Figure 7B. Compared with Group E, the variety of non-volatile flavor substances in Group $G$ increased by two times, mainly due to the addition of millet enzyme fermentation liquid, which greatly ameliorated the flavor of yogurt. Compared with Group E, the non-volatile flavor substances produced by Group $G$ were increased by 37 and reduced by 44. Among them, 2-Phenylethanol was increased 3169.6 times, a colorless liquid organic matter with a rose aroma (44). The content of hesperetin has been increased by 228.36 times, which was a natural flavonoid compound with antioxidant effect and was widely used in fruits, flowers and food (48). The hesperetin in millet yogurt is mainly derived from the added millet enzymatic fermentation broth, which is also consistent with the high antioxidant capacity of the previous yogurt. N-Acetylornithine is an intermediate from L-glutamic acid to L-arginine enzymatic biosynthesis, and the content was increased 271.22 times. L-methionine can be used in synthetic vitamins, which can protect the liver, and it is an essential 

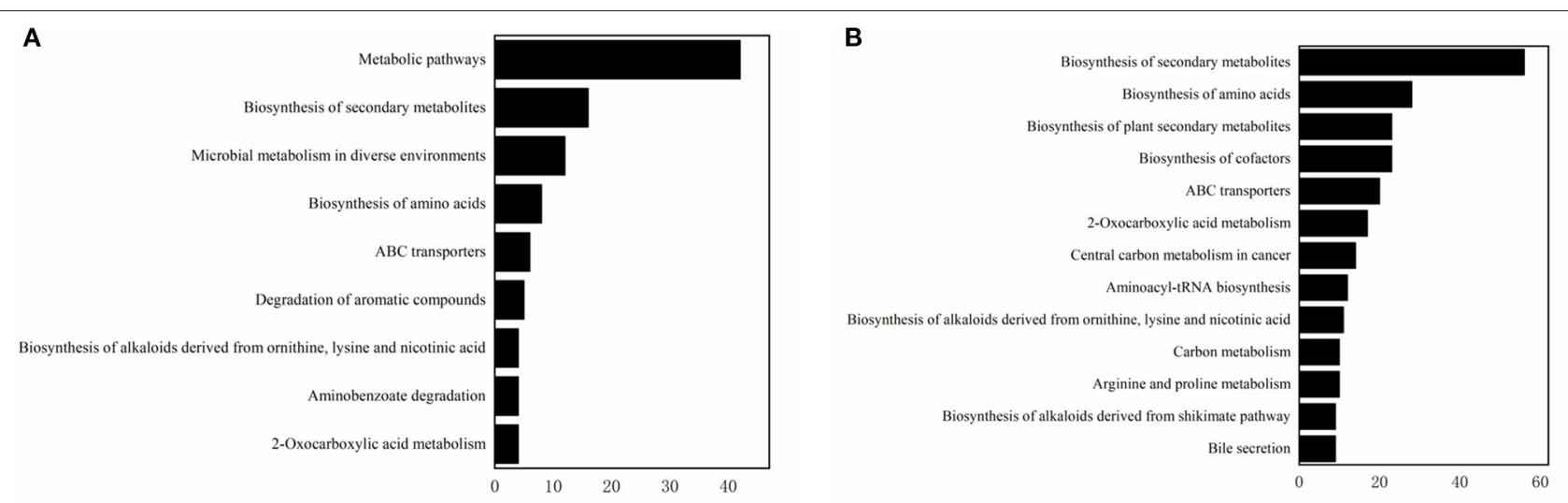

FIGURE 7 | The enriched pathways. (A) The enriched pathways involved by significantly increased metabolites when compared group F to group E. (B) The enriched pathways involved by significantly increased metabolites when compared group G to group E.

amino acid, which has significant value to the human body (49). L-methionine was increased 55.67 times in non-volatile metabolites. Most flavored compounds in yogurt were because of creamy fat decomposition and microbial transformation of lactose and citrate. The addition of millet enzymatic hydrolysis fermentation broth to yogurt enriched the variety of flavoring substances in yogurt, and this practice could also counteract the natural sourness of yogurt. This was consistent with previous reports that the addition of plant or fruit components might add sweetness or nutritional value of yogurt (50). Compared with ordinary yogurt, adding F8 and millet could improve the flavor and yogurt nutrients. The results of this part of the experiment provide insights into the metabolic mechanisms of Lactiplantibacillus plantarum subsp. plantarum F8, mainly related to the biosynthesis of secondary metabolites, amino acid metabolism and nucleotide metabolism. As well as the fingerprinting of the effect of millet enzymatic digest on nonvolatile flavor substances in yogurt represents a real advance in the study of metabolites in yogurt.

\section{Analysis of Volatile Flavor Compounds in Yogurt by HS-SPME-GC-MS}

PCA exhibited multiple components with large-to-small contributions (PC1, PC2, PC3...). 3D PCA used the first three dimensions for mapping to observe the distribution of samples in three-dimensional space. As indicated in Figure 8, the sample repeatability of the three yogurt groups was good, and the differences among the groups were large. The distance between group $\mathrm{E}$ and group $\mathrm{F}$ was relatively close, indicating that the addition of Lactiplantibacillus plantarum subsp. plantarum F8 affected the volatile flavor compounds of the two yogurts. In contrast, the distance among group G, group F and group E were far, indicating that the amount of added millet remarkably contributed to the volatile flavor of yogurt.

From Figure 9, it can be seen that 54 volatile flavor substances were found in yogurt, including alcohols, lipids, organic acids and hydrocarbons. Compared with group E, 12 volatile flavor substances were upregulated, and seven

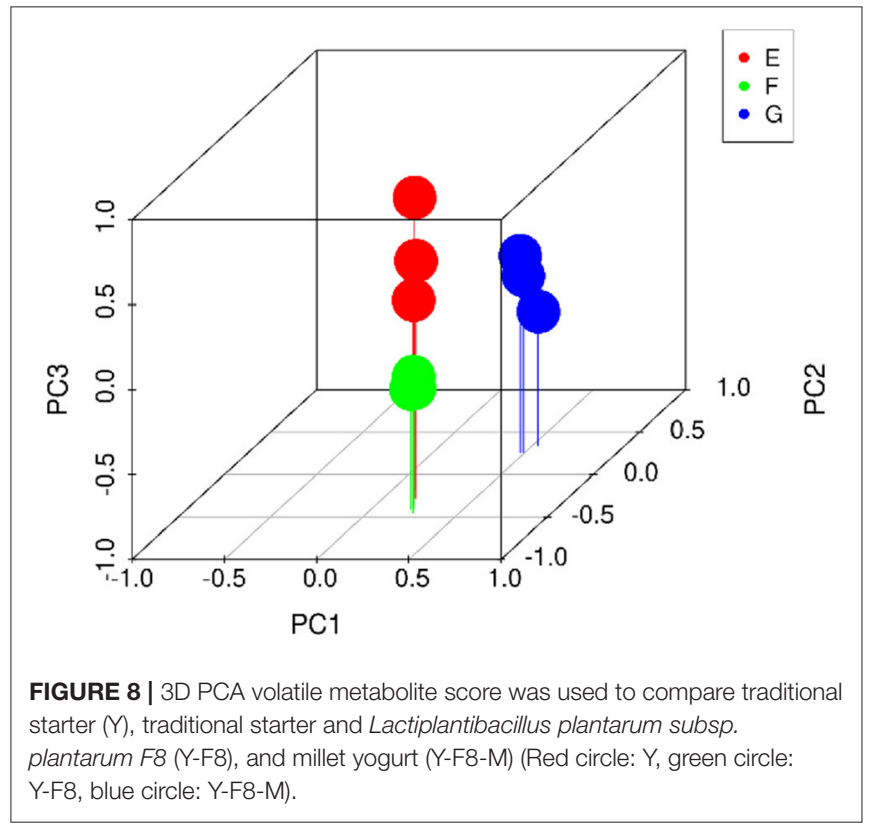

were downregulated in group F. Among these volatile flavor substances, Nonanoic acid was produced in Lactiplantibacillus plantarum subsp. plantarum via the fatty acid metabolic pathway and has a fruity flavor (3). Nonanoic acid was also detected and significantly upregulated when Tian studied the effect of four kinds of probiotic LAB on fermented milk flavor substances (51). This indicated that Nonanoic acid might be a typical flavor substance produced by probiotic LAB fermented yogurt. Oxalic acid, butyl propyl ester was produced by esterification in Lactiplantibacillus plantarum subsp. plantarum and has an aromatic odor, which was an important component of yogurt aroma (3). 2-Hydroxy-3-pentanone was produced by the conversion of glycerol by Lactiplantibacillus plantarum subsp. plantarum through the glycolytic pathway and was commonly used in food flavoring agents (52). Benzaldehyde is an organic 


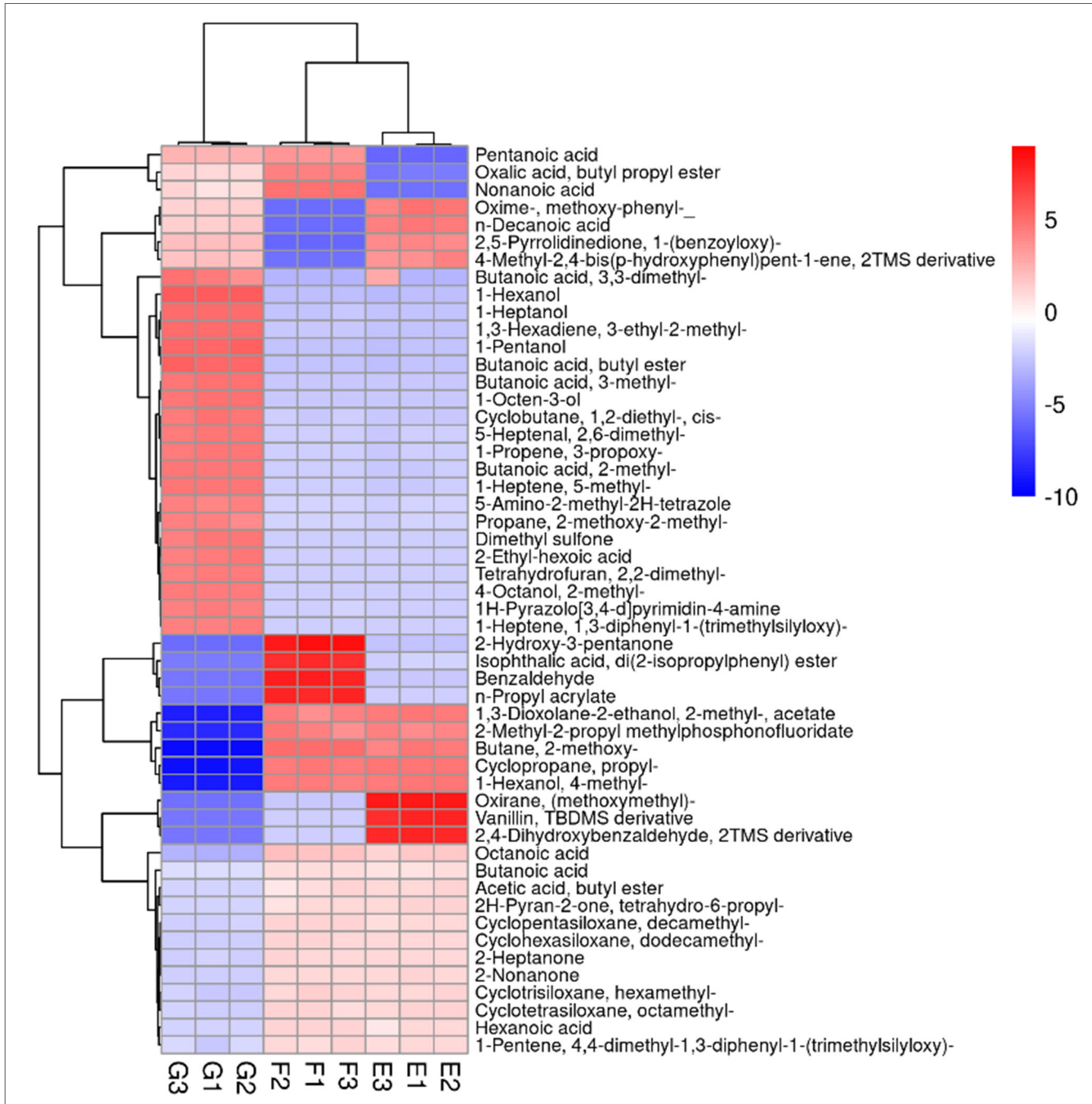

FIGURE 9 | Heat-map and hierarchical clustering of volatile metabolite profiles in yogurt. The normalized volatile metabolite abundance was visualized by color: red indicates the highest values, and blue indicates the lowest values (E: Y, F: Y-F8, G: Y-F8-M).

compound formed when the hydrogen of benzene is replaced by an aldehyde group. It was the most commonly used aromatic aldehyde with a bitter almond, cherry and nutty flavor (53). The current methods of producing benzaldehyde were mainly chemical synthesis or extraction from plants (51). This strain of Lactiplantibacillus plantarum subsp. plantarum produced benzaldehyde at relatively high levels and might have the potential to produce benzaldehyde using microbial fermentation.
Therefore, the addition of Lactiplantibacillus plantarum subsp. plantarum F8 might have a particularly positive effect on the abundance of volatile flavor substances in yogurt.

Compared with group F, 25 volatile flavor substances were upregulated in group G, of which the content of 5-Heptenal, 2,6dimethyl (with grassy, sweet, cucumber and watermelon aroma), 2-Ethyl-hexoic acid (generally used in barbecues, beverages and sweets and naturally found in wine and beer), 2-Ethyl-hexoic acid 
(slightly smelly, used for synthetic spices), 1-Hexanol (with fruity aroma), Butanoic acid, butyl ester (with fresh sweet fruit aroma, similar to banana and pineapple flavor), 1-Pentanol (slightly fruity), 1-Heptanol (with fresh and light oily fat aroma, similar to whiskey and wine-like aroma), n-Decanoic acid (with a rancid taste), Butanoic acid, and 3-methyl (with a strong cheese aroma) was significantly increased $(17,54,55)$. The formation of yogurt flavor is a complex and dynamic biochemical process (56). Millet enzymatic fermentation broth were added to enrich the volatile flavor substances in yogurt, mask the production of unpleasant volatile flavor substances, counteract the natural sour taste in yogurt, and make the product more in line with consumer taste. The addition of Lactiplantibacillus plantarum subsp. plantarum F8 and the plant-based ingredient millet may make an essential contribution to the flavor of yogurt. Additionally, they promote the formation of volatile metabolites that positively influence the aroma quality of yogurt samples (57). The results of this work provide novel knowledge about the contribution of the isolated strains and the millet enzymatic digests to the flavor profile of yogurt, which will help improve the sensory features of the final product.

\section{CONCLUSIONS}

In this study, LAB, namely, Lactiplantibacillus plantarum subsp. plantarum F8, was screened, with a SOD yield of $2476.21 \pm 1.52 \mathrm{U} \mathrm{g}^{-1}$. Meanwhile, this strain exhibited great potential as a probiotic. When this novel yogurt was used in the millet-based yogurt fermentation, its SOD activity was $19.827 \pm 0.323 \mathrm{U} \mathrm{mL}^{-1}$, higher than Bright Dairy 1911, Grand Park Old Yogurt and Weigang Flavored Yogurt Ordinary Yogurt. The addition of Lactiplantibacillus plantarum subsp. plantarum F8 significantly upregulated 12 non-volatile metabolites in yogurt, and the addition of millet enzymatic fermentation increased the non-volatile metabolites in yogurt. Thirty-seven species were significantly upregulated, including 2Phenylethanol, Hesperetin, N-Acetylornithine and L-methionine

\section{REFERENCES}

1. Macnaughtan J, Figorilli F, García-López E, Lu H, Jalan R. A double-blind, randomized placebo-controlled trial of probiotic lactobacillus casei shirota in stable cirrhotic patients. Nutrients. (2020) 12:1651. doi: 10.3390/nu12061651

2. Binda S, Hill C, Johansen E, Obis D, Ouwehand AC. Criteria to qualify microorganisms as "probiotic" in foods and dietary supplements. Front Microbiol.(2020) 11:1662. doi: 10.3389/fmicb.2020.01662

3. Aryana KJ, Olson DW. A 100-year review: yogurt and other cultured dairy products. J Dairy Sci. (2017) 100:9987-10013. doi: 10.3168/jds.2017-12981

4. Wu Z, Chen T, Pan D, Zeng X, Guo Y, Zhao G. Resveratrol and organic selenium-rich fermented milk reduces D-galactose-induced cognitive dysfunction in mice. Food Funct. (2021) 12:1318-26. doi: 10.1039/D0FO02029J

5. Aguirre-Ezkauriatza EJ, Galarza-Gonzalez MG, Uribe-Bujanda AI, RiosLicea M, Lopez-Pacheco F, Hernandez-Brenes CM, et al. Effect of mixing during fermentation in yogurt manufacturing. J Dairy Sci. (2008) 91:4454-65. doi: $10.3168 /$ jds.2008-1140

6. Borgstahl GE, Oberley-Deegan RE. Superoxide Dismutases (SODs) and SOD Mimetics. Antioxidants. (2018) 7:156. doi: 10.3390/antiox7110156 were upregulated by $3169.6,228.36,271.22$, and 55.67 times, respectively. The addition of Lactiplantibacillus plantarum subsp. plantarum F8 had a specific positive effect on the abundance of volatile flavor substances in yogurt. The addition of millet enzymatic fermentation broth enriched the volatile flavor substances in yogurt. The production of unpleasant volatile flavor substances was masked, making the product more in line with consumer taste.

\section{DATA AVAILABILITY STATEMENT}

The original contributions presented in the study are included in the article/supplementary material, further inquiries can be directed to the corresponding author/s.

\section{AUTHOR CONTRIBUTIONS}

XF: writing-original draft, data curation, investigation, methodology, and formal analysis. XL: conceptualization, writing-review and editing, data curation, formal analysis, investigation, and project administration. DP: conceptualization, project administration, funding acquisition, validation, and supervision. TZ: data curation, methodology, and supervision. ZW: formal analysis, visualization, resources, and methodology. YG: resources and methodology. XZ: conceptualization and project administration. ZS: conceptualization and formal analysis. All authors contributed to the article and approved the submitted version.

\section{FUNDING}

This work was financially supported by Natural Science Funding of China (31972048), Jiangsu Agricultural Science and Technology Innovation Fund [CX(18)3036], Jiangsu Science and Technology Department (BE2018397), the Scientific Research Foundation of Graduate School of Ningbo University (IF2021084).
7. Wang CY, Wu SJ, Shyu YT. Antioxidant properties of certain cereals as affected by food-grade bacteria fermentation. J Biosci Bioeng. (2014) 117:44956. doi: 10.1016/j.jbiosc.2013.10.002

8. Marazza JA, Nazareno MA, Giori G, Garro MS. Enhancement of the antioxidant capacity of soymilk by fermentation with Lactobacillus rhamnosus. J Funct Foods. (2012) 4:594-601. doi: 10.1016/j.jff.2012.03.005

9. Kang YM, Lee BJ, Kim JI, Nam BH, Cha JY, Kim YM. Antioxidant effects of fermented sea tangle (Laminaria japonica) by Lactobacillus brevis BJ20 in individuals with high level of $\gamma$-GT: A randomized, double-blind, and placebo-controlled clinical study. Food Chem Toxicol. (2012) 50:1166-9. doi: 10.1016/j.fct.2011.11.026

10. Xiaolong J, Baixiang P, Hehui D, Bingbing C, Hui N, Yizhe Y. Purification, structure and biological activity of pumpkin polysaccharides: a review. Food Rev Int. (2021). doi: 10.1080/87559129.2021.1904973. [Epub ahead of print].

11. Jhan F, Shah A, Gani A, Ahmad M, Noor N. Nano-reduction of starch from underutilised millets: Effect on structural, thermal, morphological and nutraceutical properties. Int J Biol Macromol. (2020) 159:1113-21. doi: 10.1016/j.ijbiomac.2020.05.020

12. Sargun M, Kiruba K, Azlin M. Physical properties of complementary food powder obtained from upcycling of Greek yogurt acid whey with kodo 
and proso millets. J Food Process Eng. (2021) 44:e13878. doi: 10.1111/jfpe. 13878

13. Song X, Sun X, Ban Q, Cheng J, Zhang S, Guo M. Gelation and microstructural properties of a millet-based yogurt-like product using polymerized whey protein and xanthan gum as thickening agents. J Food Sci. (2020) 85:3927-33. doi: 10.1111/1750-3841.15504

14. Steinkraus KH. Classification of fermented foods: worldwide review of household fermentation techniques. Food Control. (1997) 8:311-31. doi: 10.1016/S0956-7135(97)00050-9

15. Chou ST, Chao WW, Chung YC. Antioxidative activity and safety of $50 \%$ ethanolic red bean extract (Phaseolus radiatus L. var Aurea). J Food Sci. (2003) 68:21-5. doi: 10.1111/j.1365-2621.2003.tb14108.x

16. Shin EC, Lee JH, Hwang CE, Lee BW, Kim HT, Ko JM, et al. Enhancement of total phenolic and isoflavone-aglycone contents and antioxidant activities during Cheonggukjang fermentation of brown soybeans by the potential probiotic Bacillus subtilis CSY191. Food Sci Biotechnol. (2014) 23:531-8. doi: 10.1007/s10068-014-0073-9

17. Chen C, Zhao S, Hao G, Yu H, Tian H, Zhao G. Role of lactic acid bacteria on the yogurt flavour: a review. Int J Food Properties. (2017) 20:S316-30. doi: 10.1080/10942912.2017.1295988

18. Erkaya TSENG, Uuml LM. Comparison of volatile compounds in yoghurts made from cowsandrsquo buffaloesandrsquo ewesandrsquo; and goatsandrsquo milks. Int J Dairy Technol. (2011) 64:240-6. doi: 10.1111/j.1471-0307.2010.00655.x

19. Gu Y, Li X, Xiao R, Dudu OE, Yang L, Ma Y. Impact of Lactobacillus paracasei IMC502 in coculture with traditional starters on volatile and non-volatile metabolite profiles in yogurt. Process Biochem. (2020) 99:61-9. doi: $10.1016 /$ j.procbio.2020.07.003

20. Ofc A, Ahc B, Hs C, Na C, Ht B. Isolation and Identification of lactobacilli from traditional yogurts as potential starter cultures. LWT. (2021) 148:111774. doi: 10.1016/j.lwt.2021.111774

21. Fessard A, Bourdon E, Payet B, Remize F. Identification, stress tolerance, and antioxidant activity of lactic acid bacteria isolated from tropically grown fruits and leaves. Can J Microbiol. (2016) 62:550-61. doi: 10.1139/cjm,-2015-0624

22. Zhang J, Cai D, Yang M, Hao Y, Zhu Y, Chen Z, et al. Screening of folateproducing lactic acid bacteria and modulatory effects of folate-biofortified yogurt on gut dysbacteriosis of folate-deficient rats. Food Funct. (2020) 11:6308-18. doi: 10.1039/D0FO00480D

23. Yin $M$, Yang D, Lai S, Yang $H$. Rheological properties of xanthanmodified fish gelatin and its potential to replace mammalian gelatin in low-fat stirred yogurt[J]. LWT Food Sci Technol. (2021) 147:111643. doi: 10.1016/j.lwt.2021.111643

24. Xiao F, Ng VK, Marta MK, Yang H. Effects of fish gelatin and tea polyphenol coating on the spoilage and degradation of myofibril in fish fillet during cold storage. Food Bioprocess Technol. (2017) 10:89-102. doi: 10.1007/s11947-016-1798-7

25. Chen L, Zhao X, Wu J E, Liu Q, Pang X, Yang H. Metabolic characterisation of eight Escherichia coli strains including 'Big Six' and acidic responses of selected strains revealed by NMR spectroscopy. Food Microbiol. (2020) 88:103399. doi: 10.1016/j.fm.2019.103399

26. Dongmo SN, Sacher B, Kollmannsberger H, Becker T. Key volatile aroma compounds of lactic acid fermented malt based beveragesimpact of lactic acid bacteria strains. Food Chem. (2017) 229:565-73. doi: 10.1016/j.foodchem.2017.02.091

27. Cheng H. Volatile flavor compounds in yogurt: a review. Crit Rev Food Sci Nutr. (2010) 50:938-50. doi: 10.1080/10408390903044081

28. Dan T, Wang D, Jin R, Zhang H, Zhou T, Sun T. Characterization of volatile compounds in fermented milk using solid-phase microextraction methods coupled with gas chromatography-mass spectrometry. J Dairy Sci. (2017) 100:2488-500. doi: 10.3168/jds.2016-11528

29. Li S, Tian Y, Jiang P, Lin Y, Yang H. Recent advances in the application of metabolomics for food safety control and food quality analyses. Crit Rev Food Sci Nutr. (2020) 2020:1-22. doi: 10.1080/10408398.2020.1761287

30. Lin CBJW, Zl A, Qin L, Xue Z, Hya C. Metabolomic analysis of energy regulated germination and sprouting of organic mung bean (Vigna radiata) using NMR spectroscopy. Food Chem. (2019) 286:87-97. doi: 10.1016/j.foodchem.2019.01.183
31. Li D, Gao C, Zhang F, Yang R, Lan C, Ma Y, et al. Seven facts and five initiatives for gut microbiome research. Protein Cell. (2020) 11:391-400. doi: 10.1007/s13238-020-00697-8

32. Chen L, Zhao X, Wu JE, He Y, Yang H. Metabolic analysis of salicylic acid-induced chilling tolerance of banana using NMR. Food Res Int. (2020) 128:108796. doi: 10.1016/j.foodres.2019.108796

33. Kudo H, Sasaki Y. Intracellular $\mathrm{pH}$ determination for the study of acid tolerance of lactic acid bacteria. Methods Mol Biol. (2019) 1887:33-41. doi: 10.1007/978-1-4939-8907-2_4

34. Gao YX, Xu B, Fan HR, Zhang MR, Li S. 1H NMR-based chemometric metabolomics characterization of soymilk fermented by Bacillus subtilis BSNK-5. Food Res Int. (2020) 138(Pt A):109686. doi: 10.1016/j.foodres.2020.109686

35. Wu Z, Wang P, Pan D, Zeng X, Guo Y, Zhao G. Effect of adzuki bean sprout fermented milk enriched in $\gamma$-aminobutyric acid on mild depression in a mouse model. J Dairy Sci. (2021) 104:78-91. doi: 10.3168/jds.2020-19154

36. Chooruk A, Piwat S, Teanpaisan R. Antioxidant activity of various oral Lactobacillus strains. J Appl Microbiol. (2017) 123:271-9. doi: $10.1111 /$ jam. 13482

37. Ryu JA, Kim E, Kim MJ, Lee S, Kim HY. Physicochemical characteristics and microbial communities in gochujang, a traditional korean fermented hot pepper paste. Front Microbiol. (2021) 11:3543. doi: $10.3389 /$ fmicb.2020.620478

38. Ragaee S, Abdel-Aal E, Noaman M. Antioxidant activity and nutrient composition of selected cereals for food use. (2006) 98:32-8. doi: 10.1016/j.foodchem.2005.04.039

39. Lili Z, Ran F, Fazheng R, Xueying MJL. Addition of buttermilk improves the flavor and volatile compound profiles of low-fat yogurt. (2018) 98:9-17. doi: 10.1016/j.lwt.2018.08.029

40. Feng QH, Jin XG, Yu JK, Yuan QS. Detection of superoxide dismutase in milk \%. Food Sci. (1994) 56-59.

41. Cline JM, Dugan G, Bourland JD, Perry DL, Stitzel JD, Weaver AA, et al. Post-irradiation treatment with a superoxide dismutase mimic, MnTnHex2-PyP5+, mitigates radiation injury in the lungs of non-human primates after whole-thorax exposure to ionizing radiation. Antioxidants. (2018) 7:40. doi: 10.3390/antiox7030040

42. Gao HX, Yu ZL, He Q, Tang SH, ZengWC. A potentially functional yogurt co-fermentation with gnaphalium affine. LWT. (2018) 91:423-30. doi: 10.1016/j.lwt.2018.01.085

43. Dan T, Ren W, Liu Y, Tian J, Chen H, Li T, et al. Volatile flavor compounds profile and fermentation characteristics of milk fermented by lactobacillus delbrueckii subsp bulgaricus. Front Microbiol. (2019) 10:2183. doi: 10.3389/fmicb.2019.02183

44. Grasso N, Alonso-Miravalles L, O’Mahony JA. Composition, physicochemical and sensorial properties of commercial plant-based yogurts. Foods. (2020) 9:252. doi: 10.3390/foods9030252

45. Yang CY, Chen CH, Deng ST, Huang CS, Lin YJ, Chen YJ, et al. Allopurinol use and risk of fatal hypersensitivity reactions: a nationwide population-based study in Taiwan[J]. JAMA Internal Med. (2015) 175:1550-7. doi: 10.1001/jamainternmed.2015.3536

46. Lubbers S, Decourcelle N, Vallet N, Guichard E. Flavor release and rheology behavior of strawberry fatfree stirred yogurt during storage. J Agric Food Chem. (2004) 52:3077-82. doi: 10.1021/jf0352374

47. Yamanaka H, Taniguchi A, Tsuboi H, Kano H, Asami Y. Hypouricaemic effects of yoghurt containing Lactobacillus gasseri PA-3 in patients with hyperuricaemia and/or gout: a randomised, double-blind, placebo-controlled study. Modern Rheumatol. (2019) 29:146-50. doi: 10.1080/14397595.2018.1442183

48. Özcelik S, Kuley E, Özogul F. Formation of lactic, acetic, succinic, propionic, formic and butyric acid by lactic acid bacteria. LWT. (2016) 73:536-42. doi: 10.1016/j.lwt.2016.06.066

49. Su N, Ren L, Ye H, Sui Y, Li J, Ye M. Antioxidant activity and flavor compounds of hickory yogurt. Int J Food Properties. (2017) 20:1894-903. doi: 10.1080/10942912.2016.1223126

50. Farag MA, Saleh HA, Ahmady E, Elmassry MM Dissecting yogurt: the impact of milk types, probiotics, and selected additives on yogurt quality. Food Rev Int.(2021). doi: 10.1080/87559129.2021.1877301. [Epub ahead of print]. 
51. Tian H, Shen Y, Yu H, He Y, Chen C. Effects of 4 probiotic strains in coculture with traditional starters on the flavor profile of yogurt. J Food Sci. (2017) 82:1693-701. doi: 10.1111/1750-3841.13779

52. Turgut T, Cakmakci S. Probiotic strawberry yogurts: microbiological, chemical and sensory properties. Probiot Antimicrob Proteins. (2018) 10:6470. doi: 10.1007/s12602-017-9278-6

53. Le Lay C, Coton E, Le Blay G, Chobert JM, Haertlé T, Choiset Y, et al. Identification and quantification of antifungal compounds produced by lactic acid bacteria and propionibacteria. Int J Food Microbiol. (2016) 239:79-85. doi: 10.1016/j.ijfoodmicro.2016.06.020

54. Santis DD, Giacinti G, Chemello G, Frangipane MT. Improvement of the sensory characteristics of goat milk yogurt. J Food Sci. (2019) 84:2289-96. doi: 10.1111/1750-3841.14692

55. Yang S, Yan D, Zou Y, Mu D, Wu J. Fermentation temperature affects yogurt quality: a metabolomics study. Food Biosci. (2021) 42:101104. doi: 10.1016/j.fbio.2021.101104

56. Tian H, Shi Y, Zhang Y, Yu H, Mu H, Chen C. Screening of aroma-producing lactic acid bacteria and their application in improving the aromatic profile of yogurt[J]. J Food Biochem. (2019) 43:e12837. doi: 10.1111/jfbc.12837

57. Das K, Choudhary R, Thompson-Witrick KA. Effects of new technology on the current manufacturing process of yogurt-to increase the overall marketability of yogurt. LWT. (2019) 108:69-80. doi: 10.1016/j.lwt.2019.03.058

Conflict of Interest: The authors declare that the research was conducted in the absence of any commercial or financial relationships that could be construed as a potential conflict of interest.

Publisher's Note: All claims expressed in this article are solely those of the authors and do not necessarily represent those of their affiliated organizations, or those of the publisher, the editors and the reviewers. Any product that may be evaluated in this article, or claim that may be made by its manufacturer, is not guaranteed or endorsed by the publisher.

Copyright (C) 2022 Fan, Li, Zhang, Guo, Shi, Wu, Zeng and Pan. This is an openaccess article distributed under the terms of the Creative Commons Attribution License (CC BY). The use, distribution or reproduction in other forums is permitted, provided the original author(s) and the copyright owner(s) are credited and that the original publication in this journal is cited, in accordance with accepted academic practice. No use, distribution or reproduction is permitted which does not comply with these terms. 